CAHIERS DE

NARRATOLOGIE

\section{Cahiers de Narratologie}

Analyse et théorie narratives

32 | 2017

Récit et argumentation, interactions, lieux et dispositifs sociaux

\title{
La construction sérielle des personnages- narrateurs dans les fictions de Jorge Volpi : un dilemme catégoriel
}

\section{Sara Calderón}

\section{OpenEdition}

\section{Journals}

Electronic version

URL: http://journals.openedition.org/narratologie/7845

DOI: 10.4000/narratologie.7845

ISSN: 1765-307X

Publisher

LIRCES

\section{Electronic reference}

Sara Calderón, «La construction sérielle des personnages-narrateurs dans les fictions de Jorge Volpi : un dilemme catégoriel », Cahiers de Narratologie [Online], 32 | 2017, Online since 21 December 2017, connection on 01 May 2019. URL : http://journals.openedition.org/narratologie/7845 ; DOI : 10.4000/ narratologie. 7845

This text was automatically generated on 1 May 2019.

Article L.111-1 du Code de la propriété intellectuelle. 


\title{
La construction sérielle des personnages-narrateurs dans les fictions de Jorge Volpi : un dilemme catégoriel
}

\author{
Sara Calderón
}

1 Un des mérites des fictions post-modernes et contemporaines a été de poser souvent à la critique littéraire et à la narratologie de nouveaux défis qui sont autant de questionnements à propos de la pertinence qu'il y aurait à une reformuler, voire à reconceptualiser certaines catégories d'analyse.

2 L'œuvre de l'écrivain mexicain Jorge Volpi a sa place dans cette mouvance car elle a désormais acquis notoriété, outre pour ses qualités d'érudition et de réflexion, pour les jeux narratifs complexes qu'elle met en place. Cependant, au delà des jeux que chacune des fictions établit avec les ressources formelles, il apparait au lecteur averti que, par delà la diversité des thématiques et des structures, l'ensemble d'entre elles finit par mettre en place la construction presque sérielle d'une sorte d'entité dont on pourrait se demander si elle relève ou non du personnage. En effet, peut-être dans un clin d'œil à Borges, dont il est un admirateur déclaré, Jorge Volpi prête dans ses romans de façon récurrente au personnage principal, qui souvent est le narrateur, son nom ou prénom, ainsi que certaines données personnelles. Néanmoins, il mène cette pratique bien plus loin que l'illustre Argentin, la lecture consécutive des romans mettant en place, de par la répétition, une entité homogène qui, excédant chacun d'entre eux, possède au final une présence propre. Cette entité, qui ne relève que du contenu latent si on prend les romans séparément, se révèle au fil des lectures, jetant des ponts entre les œuvres. Outre le fait qu'elle a un impact indéniable sur l'activité de lecture du lecteur capable de l'actualiser, elle est à la source de nombreux questionnements du point de vue de la catégorisation critique, car son étude remet en cause certains des outils d'analyse les plus établis. Il est notre propos d'examiner ici la façon dont cette entité se construit et les implications que 
cette construction recouvre tant du point de la lecture des fictions de Volpi que de leur analyse.

3 C'est dès son premier roman que Jorge Volpi assoit la base de ce qui peut apparaître maintenant comme une sorte de construction sérielle. En effet, A pesar del oscuro silencio (1992) rapporte l'histoire d'un universitaire, Jorge, qui entame l'étude de l'œuvre du célèbre poète Jorge Cuesta et finit par s'identifier tellement à lui qu'il en vient à reproduire sa vie, comme gagné par son inquiétante présence. Le roman entier repose sur le jeu identitaire qui se met en place entre Jorge Cuesta, Jorge le narrateur et l'auteur Jorge Volpi. En effet, non seulement certains des amis écrivains de Volpi se retrouvent dans la fiction en tant qu'amis du narrateur, mais en outre Jorge a écrit un essai sur Jorge Cuesta qui porte le titre d'un des articles de Volpi. Par ailleurs, la première édition du roman pousse le jeu jusqu'à intégrer dans la première de couverture une photo de Jorge Volpi adoptant la pose d'une des photos les plus connues de Jorge Cuesta. C'est donc le roman entier qui repose sur la perception d'une fluctuation identitaire pour faire émerger un sentiment d'inquiétant étrangeté. Ce premier personnage narrateur, que l'on pourrait presque considérer fondateur, peut ainsi être pensé comme base de ce qui deviendra par la suite une configuration récurrente. Avant d'en isoler les caractéristiques communes, il est possible d'examiner brièvement les quelques personnages dont elle se dégage par recoupement.

4 Toutefois il faut signaler que, sans répondre encore tout à fait à la caractérisation qui sera systématisée par la suite, les personnages de La paz de los sepulcros (1995) et de El temperamento melancólico (1996) ont en commun avec les autres leur démesure et leur manque de scrupules. Alors que dans le premier le journaliste Agustín Oropeza est prêt à tout pour avoir une exclusive, dans le contexte politique très noir que fut celui du Mexique en 1994, Carl Gustav Gruber est un réalisateur de cinéma moribond prêt à sacrifier toute sa troupe d'acteurs pour filmer au plus proche du réel dans ce qu'il espère son dernier chef-d'œuvre.

5 Reprenant la suite d'une série bien plus régulière, Gustav Links, le mathématicien et narrateur de En busca de Klingsor (2000) est un homme d'âge mûr, sommé d'aider le jeune lieutenant Francis $\mathrm{P}$. Bacon dans son enquête à propos de l'identité secrète de Klingsor, le conseiller du Führer dans les questions concernant la science. La narration s'effectue de son point de vue, qui phagocyte l'ensemble du monde fictionnel ne laissant sur lui aucune autre prise. Elle dévoile que Gustav Links est un criminel : le lecteur est certain qu'il est le responsable de la mort de Natalia, la femme qu'il aime, pour avoir dénoncé l'Opération Walkyrie, dans laquelle son mari était impliqué. Le roman laisse également penser qu'il pourrait être Klingsor lui-même.

6 Dans El fin de la locura (2003), le psychanalyste Aníbal Quevedo suit, dans le cadre du Paris de mai 68, un apprentissage auprès de certains des intellectuels de premier ordre à l'époque : Jacques Lacan, Louis Althusser. Par la suite, il rentre au Mexique, où il finit par s'installer dans une proximité et une complicité avec le pouvoir politique qui l'éloigne de ses prétendus idéaux. Au cours de son cheminement, Aníbal Quevedo apparaît comme un personnage opportuniste, voire immoral, tant dans ses engagements politiques que dans son attitude envers Claire, la femme qu'il aime et avec laquelle il entretient une relation tourmentée.

7 Le journaliste Yuri Chernishevski est de son côté le personnage-narrateur dans No será la tierra (2006). Le roman rapporte le moment de la chute de l'URSS, tout en faisant état des avancées scientifique dans la course, scientifique et commerciale, aux découvertes sur 
l'ADN. Il mêle pour ce faire l'histoire de vie de plusieurs femmes actives et charismatiques. Yuri Chernishevski tombera amoureux de l'une d'entre elles, la biologiste de prodige Eva Hálasz, et vivra également avec elle une histoire d'amour tourmenté. C'est d'ailleurs sa jalousie qui l'amènera à finalement tuer aussi sous ses coups, dans un accès de furie, la femme qu'il aime.

Le narrateur de El jardín devastado (2008) est également un intellectuel, en ce cas un professeur universitaire en sciences politiques, à qui on demande de faire une étude historique et politique sur l'Irak de la guerre du Golfe. Néanmoins, le roman n'approfondit pas comme les autres dans le contextuel, et reste très intimiste, se concentrant sur le drame humain de sa protagoniste, Leila, et sur les affres du narrateur, que l'on suppose inspirées de la vie de Volpi lui-même. Comme les autres personnagesnarrateurs, celui-ci a une relation très complexe et tourmentée à sa compagne.

Finalement, l'action de Memorial del engaño (2006) se déroule dans les milieux financiers, tant des négociations ayant conduit à la création du FMI pendant la Guerre Froide que des spéculations ayant entraîné la crise de 2008. Son personnage principal et narrateur J. Volpi est un génie des finances sans scrupules qui est en fuite pour avoir détourné 15 millions de dollars. Sa vie privée révèle également un monstre qui utilise ses propres enfants, traite avec un mépris sans limites son ex-femme et mène une double vie avec sa femme et son amant.

$10 \mathrm{Au}$ fil des romans, une série de traits se répètent chez ces personnages. Certains seront sans doute déjà apparus au lecteur, on les énumérera brièvement. Le premier trait qui lie les personnages entre eux ainsi qu'à Jorge Volpi est l'écriture : si, comme nous l'avons dit Jorge est l'auteur d'un essai portant le titre d'un des articles de Volpi, la plupart des autres narrateurs sont les auteurs dans la fiction du livre que le lecteur a dans les mains. C'est ce qui se passe avec En busca de Klingsor (2000), El fin de la locura (2003), No será la tierra (2006), El jardín devastado (2008), Memorial del engaño (2013).

11 D'autre part, par delà leurs différences contextuelles, ces narrateurs partagent également des traits de caractère : un degré de perversion plus ou moins accentué qui va de pair avec une ambition plus ou moins poussée, un certain égoïsme et un besoin important de contrôle. Ainsi, ces narrateurs ne sont pas des modèles de vertu. Leur psychisme va de l'opportuniste sans scrupules qu'est Aníbal Quevedo (Volpi, 2003) jusqu'au monstre sans remords qu'est J. Volpi (Volpi, 2013) en passant par toute une série de caractérisations intermédiaires. Les narrateurs portent souvent le nom ou le prénom de Volpi, plus ou moins déguisé, comme dans No será la tierra (2006), où Yuri est l'équivalent russe de Jorge. D'autre part, ils peuvent aussi se dédoubler : c'est ce qui se passe dans El fin de la locura (2003), où l'éditeur Jorge Volpi et le psychanalyste Aníbal Quevedo se partagent les traits de cette entité. C'est également ce qui se produit dans El temperamento melancólico (1996) où, bien que ne portant pas le nom ou le prénom de Volpi, le personnage de Javier et celui de Carl Gustav Gruber, se partagent eux aussi les caractéristiques de l'entité.

12 Associée a ce profil psychologique, une condition existentielle est également récurrente chez ces personnages-narrateurs : comme il a été dit, ils ont souvent été à l'origine de la mort ou du malheur des femmes qu'ils aiment, voire de leurs êtres chers ou de toute une série de personnes, comme J. Volpi (Volpi, 2013) ou Gustav Links (Volpi, 2000). De ce fait, ils vivent fréquemment au moment de la narration dans la solitude et le désenchantement. Ce sont presque toujours l'égoïsme et le besoin de contrôle qui les y ont conduit. 

cette entité. Ce quasi-personnage sujet à variantes introduit une dimension esthétique, constituant tout d'abord un clin d'œil aux accents postmodernes qui vient s'ajouter aux jeux permanents entre le fictionnel et le réel qui parsèment les romans de Volpi. En effet, bien que la critique situe souvent Jorge Volpi dans les post-postmodernes (Williams, Rodríguez, 2002: 153), il est évident que de nombreuses caractéristiques de ses romans seraient impensables sans l'héritage reçu de la postmodernité. Pour ce qui nous occupe, les constructions sérielles et le décloisonnement des frontières sont deux des caractéristiques évidentes associées aux manifestations culturelles postmodernes. Ainsi, à propos de l'œuvre de Warhol, Fredric Jameson signalait la marchandisation, qui met en avant la fétichisation des produit, la naissance d'une nouvelle sorte de superficialité ainsi que d'une esthétique qui révèle le substrat décharné des objets sous leurs apparences colorées et une disparition de l'être humain en faveur de son image comme caractéristiques de la postmodernité (Jameson, 1995: 28-32). D'autre part, le fait que Volpi choisisse de faire de son personnage un être le plus souvent machiavélique s'insère bien aussi dans cette mouvance, où souvent les points de vue excentrés ont été privilégiés et dans laquelle l'exploitation du point de vue du monstre a opéré en tant qu'élément de rénovation de nombreux genres littéraires ${ }^{1}$. Le choix d'élaborer de façon sérielle une entité machiavélique situe donc bien lui aussi la production de Jorge Volpi dans son époque et introduit dans ses romans une dimension esthétique inattendue.

Cependant, l'entité ainsi mise en place va très largement au delà de l'empreinte esthétique d'une époque et finit par conformer par la réitération une sorte de surplus de sens qui n'est pas sans susciter, tant du point de vue de la narration que de la réception, des questionnements qu'il faut s'atteler à résoudre. Puisque c'est de la répétition que se dégage une homogénéité, l'entité demeure dans chacun des romans en tant que contenu latent actualisable uniquement en fonction de la connaissance encyclopédique que peut avoir le lecteur de l'œuvre de Jorge Volpi. Néanmoins, pour peu que ce contenu puisse être actualisé, et cela peut se produire dès la lecture attentive de deux romans, il est impossible qu'il demeure sans impact sur la lecture.

Raphaël Baroni faisait état dans La tension narrative. Suspense, curiosité et surprise (2007) de la façon dont les théories post-structuralistes de la narration avaient accentué le développement de perspectives axées sur la réception ainsi que de l'étude des contextes. En ce sens, des catégories telles que les genres littéraires, jusqu'alors envisagées surtout pour leur valeur descriptive et classificatoire avaient été approchées sous l'angle de la réception et $\mathrm{du}$ contexte. Baroni signalait ainsi que, sous cette perspective, le genre littéraire inscrivait l'interprétation dans un contexte historique et social et devenait également un guide d'écriture ou de lecture. (Baroni, 2011 : 225-226).

Sans ressortir du genre littéraire, l'entité qui se configure au fil des romans de Volpi n'en demeure pas moins une structure qui rattache entre eux tous ces romans, du fait de la stabilité relative qu'on a évoquée. Cela d'autant plus qu'il s'agit d'une stabilité qui va au delà de la caractérisation pure et simple du personnage pour concerner aussi une partie de ses agissements et du schéma actantiel, avec le rapport destructeur que ces narrateurs établissent à leurs proches. De ce fait, si le lecteur actualise l'entité, qui parce qu'elle agit et qu'elle concerne le schéma actantiel est aussi une structure, celle-ci opère en tant que régularité narrative qui informe la lecture : le lecteur s'attendra à voir quelle forme elle adopte, jusqu'où va son degré de perversité etc. L'entité ainsi conformée en régularité a donc un double impact. D'une part, elle agit en tant que schéma de lecture, balisant 
l'actualisation du roman par le lecteur comme pourraient le faire les conventions d'un genre marqué par des conventions assez visibles, comme le genre policier ou le roman d'espionnage. D'autre part, elle investit le texte d'une charge thymique supplémentaire. En effet, elle nous semble introduire quelque chose qui serait de l'ordre à la fois du suspense paradoxal et du plaisir du retour d'un élément connu évoqués par Baroni. Le narratologue expliquait que lorsqu'une anticipation se met en place chez le lecteur à la lecture d'œuvres rattachées à des genres littéraire très codés, la tension dérivée de l'incertitude se déplace du « qu'est-ce qui va se passer? » à « quand ? » ou « comment? » cela va se passer (Baroni, 2001 : 276-277). Ainsi, bien qu'une partie de l'histoire puisse être anticipée avec une certaine marge de certitude, le suspense demeurait. Puis, il expliquait également que, même dans les cas de totale répétition, lors d'une relecture par exemple, la charge émotionnelle pouvait perdurer, mais elle tenait alors au plaisir du retour d'un élément connu (Baroni, 2011 : 288-295).

La présence dans les romans de Volpi de cette entité, qui subsiste par delà les changements contextuels et de fond, produit donc de réels effets de lecture, tant du point de vue de l'appréhension rationnelle du contenu que de la charge thymique du récit. Cependant, cette combinaison de virtualité et d'indéniable présence finit, il est vrai, par susciter de nombreux questionnements quant à la façon d'aborder d'un point de vue critique l'entité qui se dégage de la répétition.

En effet, la première question qui se pose est celle de son statut. C'est précisément dans la capacité à configurer des personnages que Dorrit Cohn situait un des traits distinctifs de la fiction: elle précisait ainsi que "le procédé principale dont se sert la fiction pour transformer le monde réel (...) consiste à en augmenter la population : elle y implante des êtres imaginaires, que nous appelons communément « personnages » " (COHN, $2001: 33$ ). S'il est bien entendu que chacun des personnages-narrateur auparavant évoqués est bien un personnage, soit un être fictif construit textuellement, il est déjà plus difficile d'assurer que l'entité qui se dégage du recoupement des traits stables de chacun d'entre eux en soit un également. Bien qu'elle ne soit présente qu'en potentialité dans chacun des textes, il serait également abusif de considérer qu'elle est de l'ordre du fantasme, car il s'agit bien d'un effet de lecture qui émerge des textes parce qu'il s'y trouvait. Compte tenue du fait que les quelques traits stables qui concernent le caractère et le parcours vital lui donnent une présence et même une " corporéité » très marquées qui émergent $\mathrm{du}$ croisement des univers fictionnels, nous proposons pour l'instant de considérer l'entité qui découle de la construction sérielle comme une sorte de personnage intertextuel, puisque c'est la connaissance encyclopédique de l'œuvre de Volpi qui détermine son actualisation.

Une deuxième question que peut soulever cette construction serait de savoir s'il faut la relier au texte ou au paratexte. En effet, bien que ressortissant à n'en pas douter d'un phénomène intertextuel, dans la mesure où la mise en place des personnages-narrateurs décloisonne les frontières entre le «dedans » et le "dehors » du texte, ce problème ne peut que se répercuter également sur l'entité. Ainsi Gustav Links (Volpi, 2000), Aníbal Quevedo (Volpi, 2003), Yuri Chernishevski (Volpi, 2006) et J. Volpi (Volpi, 2013) sont explicitement les auteurs du volume dans la fiction. Le titre y est explicitement donné, parfois la démarche est poussée jusqu'à faire coïncider également la date et la maison d'édition avec celles du volume réel que le lecteur a entre ses mains. Dans El jardín devastado (2008), le narrateur est également supposé être l'auteur du volume. Dans 
Memorial del engaño (2013) c'est tout le paratexte qui est fictionnel, les couvertures et quatrièmes de couverture du livre contenant la biographie du personnage.

Outre sa nature intertextuelle, l'entité se situe donc entre le texte et le paratexte en ce sens que la plupart des personnages qui l'intègrent jettent des ponts entre les deux, soit parce que le roman fait allusion à un paratexte fictionnel, soit parce que les données du volume fictionnel et celles du volume réel coïncident, soit encore parce que le texte colonise la place auparavant réservé au paratexte. Cette situation frontalière renforce par ailleurs le mélange entre le réel et le fictionnel qui était déjà à l'origine des personnages, avec l'intégration de données personnelles du narrateur à ces constructions textuelles. Bien que, comme il a été dit, le décloisonnement des frontières ait été récurrent dans la production postmoderne, il faut signaler ici que dans le cas des fictions de Volpi il est double : d'une part la création d'une projection fictionnelle de l'écrivain; d'autre part la colonisation par la fiction de «l'extérieur » du livre qui se produit soit avec la coïncidence des données des volumes, soit avec des phénomènes plus explicites tels que la photo de la première édition de A pesar del oscuro silencio (1992) ou encore l'insertion dans El fin de la locura (2003) d'une bibliographie semi-fictionnelle intégrant des œuvres portant sur le personnage, soit avec des phénomènes qu'on pourrait qualifier de massifs, avec la totale colonisation de la couverture dans Memorial del engaño (2013).

21 L'entité qui se dégage de la charge auto-intertextuelle que peut apporter la répétition lors des lectures des différents romans de Volpi est latente dans chacun d'entre eux, présente en sa virtualité. Il est difficile d'assurer que sa présence ne se soit pas faite de façon non préméditée à la suite de ce qui avait commencé comme un de nombreux jeux narratifs dont Jorge Volpi est adepte, mais sa présence aujourd'hui dans une œuvre déjà conséquente est indéniable. Outre le fait qu'elle a des incidences dans l'opération de lecture que met en place le lecteur chevronnée de l'œuvre de l'auteur mexicain, elle remet par ailleurs profondément en question plusieurs des catégories d'analyse élaborées par la critique pour l'étude de la production littéraire.

22 L'entité agit en effet du point de vue de la lecture d'une façon analogue au balisage que mettrait en place une structure du type d'un genre littéraire relativement codé : elle balise, facilite l'interaction du lecteur avec le texte et investit celui-ci d'une charge thymique supplémentaire redevable de la seule présence de cette entité. Du point de vue de l'esthétique, elle constitue à n'en pas douter un clin d'œil à la société de consommation et à la culture populaire qui intègre bien le mélange culture savante - culture populaire que, d'un certain point de vue, l'on peut retrouver dans les œuvres de l'écrivain mexicain avec le contenu de divulgation scientifique, historique, économique... qu'elles comportent souvent.

Du point de vue des outils d'analyse critique, l'entité se profile également comme un défi qui s'insère bien dans la relation de provocation que Jorge Volpi, et tout le groupe d'auteurs Crack avec lesquels son nom est apparu sur la scène internationale, entretient à sa critique. Non en vain l'auteur s'engageait par boutade dans son "Código de procedimientos litterarios del Crack » à " réserver aux critiques littéraires le traitement que l'on donne aux êtres humains $»^{2}$. Néanmoins, ces jeux textuels peuvent être aussi envisagés comme la pointe d'un plus vaste iceberg, avec le phénomène des écrivains, également enseignants en littérature, qui jouent, sinon un double jeu, du moins un jeu où ils sont en possession des armes de deux camps. Quoiqu'il en soit, il s'agit de notre point de vue d'un jeu salutaire qui amènera peut-être à terme s'il se poursuit critiques et narratologues à renouveler les outils d'analyse dont ils disposent aujourd'hui. 


\section{BIBLIOGRAPHY}

BARONI, Raphaël, La tension narrative. Suspense, curiosité et surprise, Paris, Ed. du Seuil, 2007.

CALDERON, Sara,

- Jorge Volpi ou l'esthétique de l'ambiguité, Paris, L'Harmattan, 2010.

- «El género policiaco como recurso narrativo en En busca de Klingsor de Jorge Volpi », Cahiers de narratologie, $\mathrm{n}^{\circ} 29$, décembre 2015. https://narratologie.revues.org/7457 (dernière consultation, mars 2016)

- «Imaginario neocolonial y técnicas de individuación : McOndo y el grupo Crack », Réseau d'études décoloniales. Actes du colloque «Etudes décoloniales : déplacements épistémologiques du pouvoir, de l'être et des savoirs ", Université Lumière Lyon 2, 7 et 8 décembre 2015. A paraître en 2016.

COHN, Dorrit, Le propre de la fiction, Paris, Seuil, 2001.

ECO, Umberto, Lector in fabula. Le rôle du lecteur, Paris, Grasset, coll. Poche, 1985.

JAMESON, Fredric, El posmodernismo o la lógica cultural del capitalismo avanzado, Barcelona, Paidos, 1995.

ROAS, David, Tras los límites de lo real. Una definición de lo fantástico, Madrid, Páginas de espuma, 2011.

WILLIAMS, Raymond L., La narrativa posmoderna en México, Xalapa, Biblioteca Universidad Veracruzana, 2002.

VOLPI, Jorge,

- A pesar del oscuro silencio, México, Seix Barral, 2001. (1ed, 1992, Joaquín Mortiz)

- El temperamento melancólico, México, Nueva Imagen, 1996.

- En busca de Klingsor, Barcelona, Seix Barral, 1999.

- El fin de la locura, Barcelona, Seix Barral, 2003.

- «Código de procedimientos literarios del Crack », dans CHÁVEZ, Ricardo ; ESTIVILL, Alejandro ; HERRASTI, Vicente ; PADILLA, Ignacio ; PALOU, Pedro Angel ; REGALADO, Tomás ; URROZ, Eloy ;

Volpi, Jorge, Crack. Instrucciones de uso, Barcelona, Mondadori, 2004.

- No será la Tierra, Madrid, Alfaguara, 2006.

- Memorial del engaño, Madrid, Alfaguara, 2013.

\section{NOTES}

1. David Roas fait état dans son étude de comment, en lien avec le nouveau paradigme de réalité et la vision postmoderne du sujet, un des ressorts de renouveau du fantastique dans la postmodernité a été d'adopter le point de vue du monstre (Roas, 2011 : 143-176).

2. Nous traduisons, « conceder a los críticos literarios el trato reservado a los seres humanos » (Chávez ; Estivill ; Herrasti ; Padilla ; Palou ; Regalado ; Volpi, 2004 : 184). 


\section{ABSTRACTS}

Les fictions post-modernes et contemporaines ont parfois amené la critique littéraire et la narratologie à s'interroger sur leurs catégories d'analyse. Située dans cette mouvance, l'œuvre de Jorge Volpi met en place de nombreux jeux narratifs. Si beaucoup de ses romans intègrent un personnage-narrateur modelé sur l'image de l'auteur, il apparaît au lecteur averti que cette construction presque sérielle aboutit lors de la lecture consécutive des romans à la mise en place d'une sorte d'entité transnarrative. Celle-ci se constitue autant en sorte de personnage-narrateur intertextuel qu'en régularité narrative, jetant des ponts entre les romans. Elle influence et paramètre ainsi l'activité de lecture du lecteur capable de l'actualiser et se situe à la source de nombreux questionnements du point de vue de la catégorisation critique, son étude remettant en cause certains des outils d'analyse les plus établis.

Las ficciones postmodernas y contemporáneas han conducido a veces a la crítica literaria y a la narratología a interrogarse sobre sus categorías de análisis. La obra de Jorge Volpi se sitúa dentro de esta dinámica con los numerosos juegos narrativos que pone en marcha. Si muchas de sus novelas integran un personaje-narrador modelado sobre la imagen del autor, rápidamente aparece que, para el lector experimentado, esta construcción casi serial desemboca al leer de forma consecutiva las novelas en la emergencia de una suerte de entidad transnarrativa. Esta se constituye tanto en suerte de personaje-narrador intertextual como en regularidad narrativa, estableciendo vínculos entre las novelas. La entidad influencia y regula así la actividad de lectura del lector capaz de actualizarla. Se sitúa en el origen de numerosos cuestionamientos desde el punto de vista de la categorización crítica, ya que su estudio pone en tela de juicio algunas de las herramientas de análisis más establecidas.

INDEX

Mots-clés: transnarrativité, personnage intertextuel, Jorge Volpi, paratexte fictionnel, récit, anticipation (ou régularité) lectoriale, jeux narratifs

\section{AUTHOR}

\section{SARA CALDERÓN}

Maître de Conférences, Université Nice Sophia Antipolis, LIRCES EA3159, Université Côte d'Azur 